 \\ MOUNTaiNS \\ Mathematical Publications
}

\section{MOMENTS OF VECTOR-VALUED FUNCTIONS AND MEASURES}

\author{
Miloslav Duchoñ — Camille Debiève
}

\begin{abstract}
There are investigated conditions under which the elements of a normed vector space are the moments of a vector-valued measure, and of a Bochner integrable function, respectively, both with values in a Banach space.
\end{abstract}

\section{Introduction}

If $g$ is a real-valued function of bounded variation on the unit interval $I$ of the real line, the numbers

$$
a_{k}=\int_{0}^{1} t^{k} \mathrm{~d} g(t) \quad(k=0,1, \ldots)
$$

are called the moments of $g, g$ being, e.g., distribution of mass of particle, body, or electric charge. Hausdorff $[\underline{\mathrm{H}}]$ has shown that for a sequence $a_{k}$ of real numbers to be moment sequence of some non-decreasing $g$ (the case of particular interest in some cases), it is necessary and sufficient that $a_{k}$ be totally monotone. Recall that a sequence is called totally monotone if all its difference sequences are non-negative.

We shall search conditions under which, given a sequence $a_{n}, n=0,1,2, \ldots$. of complex numbers,

(a) there exists a measure on $[a, b]$ such that $a_{n}$ are the moments of the measure, respectively,

(b) there exists a function in $L_{p}([a, b]), 1 \leq p \leq \infty$, such that $a_{n}$ are the moments of this function.

2000 Mathematics Subject Classification: 44A60, 28 B99.

Keywords: Banach space, vector valued measure, Bochner integral, moments of measures and functions.

Supported by the Slovak grant agency VEGA, grant number 2/4137/07. 


\section{MILOSLAV DUCHOŇ — CAMILLE DEBIÈVE}

Then, in Section $3, a_{n}$ are taken to be elements of a normed vector space and conditions are given under which $a_{n}$ are the moments of (a) a vector-valued measure, and (b) of a Bochner integrable function, respectively.

\section{Preliminaries}

The Hausdorff moment problem $[\underline{\mathrm{H}}, \mathrm{LO}, \mathrm{W}]$ is the following: given a prescribed set of real numbers $\left\{a_{n}\right\}_{0}^{\infty}$, find a bounded non-decreasing function $u(t)$ on the closed interval $[0,1]$ such that its moments are equal to the prescribed values, that is,

$$
\int_{[0,1]} t^{n} \mathrm{~d} u(t)=a_{n}, \quad n=0,1,2, \ldots
$$

The integral is a Riemann-Stieltjes integral. Equivalently, find a nonnegative measure $\mu$ on borelian subsets in $[0,1]$ with

$$
\int_{[0,1]} t^{n} \mathrm{~d} \mu(t)=a_{n}, \quad n=0,1,2, \ldots
$$

We shall need the operator $\nabla^{k}(k=0,1,2, \ldots)$ defined by

$$
\begin{gathered}
\nabla^{0} a_{n}=a_{n}, \quad \nabla^{1} a_{n}=a_{n}-a_{n+1}, \\
\nabla^{k} a_{n}=a_{n}-\left(\begin{array}{c}
k \\
1
\end{array}\right) a_{n+1}+\left(\begin{array}{c}
k \\
2
\end{array}\right) a_{n+2}-\cdots+(-1)^{k} a_{n+k}, \quad n=1,2, \ldots
\end{gathered}
$$

for any sequence of real or complex numbers $\left\{a_{n}\right\}_{0}^{\infty}$.

Remark that we confine ourselves to interval $[0,1]$ for simplicity, this is, however, no limitation of generality, for any bounded interval $[a, b]$.

Definition. For each $f \in L_{1}([0,1])$, the elements

$$
a_{n}=\int_{0}^{1} t^{n} f(t) \mathrm{d} t, \quad n=1,2, \ldots
$$

are called the moments of $f$. For a measure $\mu$ on $[0,1]$, the elements

$$
a_{n}=\int_{0}^{1} t^{n} \mu(\mathrm{d} t), \quad n=0,1,2, \ldots
$$

are called the moments of $\mu$. 


\section{MOMENTS OF VECTOR-VALUED FUNCTIONS AND MEASURES}

For a given sequence $a=\left\{a_{n}\right\}_{0}^{\infty}$, put

$$
l_{k, m}=\left(\begin{array}{c}
k \\
m
\end{array}\right) \nabla^{k-m} a_{m}, \quad(k, m=0,1,2, \ldots) .
$$

Let us mention that this definition is sligthly different from the definition of $\lambda_{k, m}$ given in [W], but we obtain similar results.

In order to remain in some reasonable frame in the following we shall suppose satisfied the

Condition A. There is a finite positive constant $L$ such that

$$
\sum_{k=0}^{N}\left|l_{N, k}\right|<L, \quad N=0,1,2, \ldots
$$

Following [W, p. 107] for the sequence $\left\{a_{n}\right\}$, define an operator $L_{N}(t)$ by

$$
L_{N}(t)=(1+N) l_{N,([N t])}, \quad N=1,2, \ldots
$$

$[N t]$ means the largest integer contained in $N t$.

We have [W, p. 110] for $p>1$,

$$
\int_{0}^{1}\left|L_{N}(t)\right|^{p} \mathrm{~d} t=\frac{(N+1)^{p}}{N} \sum_{m=0}^{N-1}\left|l_{N, m}\right|^{p}<2 L, \quad N=0,1,2, \ldots
$$

If $f$ is in $L_{p}([0,1])$ and $a_{n}$ are its moments, then [W, p. 111]

$$
\lim _{N \rightarrow \infty} \int_{0}^{1} t^{n} L_{N}(t) \mathrm{d} t=a_{n}, \quad n=0,1, \ldots
$$

Hence for every continuous, and more generally, for every bounded measurable function $g$ there exists

$$
\lim _{N \rightarrow \infty} \int_{0}^{1} g(t) L_{N}(t) \mathrm{d} t=A .
$$

If $a_{n}$ are moments of $f$, then for all $t \in[0,1]$, we have

$$
\int_{0}^{1}\left|L_{N}(s)\right| \mathrm{d} s \leq \int_{0}^{1} K_{N}(t, s)|f(s)| \mathrm{d} s
$$

where

$$
K_{N}(t, s)=(1+N)\left(\begin{array}{c}
N \\
{[N t]}
\end{array}\right) s^{[N t]}(1-s)^{N-[N t]}, \quad(N=1,2 \ldots ; 0 \leq s, t \leq 1) .
$$

It is easy to prove the following lemma. 
Lemma 1. For every $t \in[0,1]$

$$
\int_{0}^{1}\left|K_{N}(t, s)\right| \mathrm{d} s \leq \frac{N+1}{N} \sum_{m=0}^{N-1} \int_{0}^{1}\left(\begin{array}{l}
N \\
m
\end{array}\right) s^{m}(1-s)^{N-m} \mathrm{~d} s \leq M<\infty,
$$

for $N=1,2, \ldots$

\section{Scalar-valued moments}

At least the part of the following theorem is already known see WW, pp. 100-114 ], where the $L_{p}$ case $(1<p \leq \infty)$ and (iii)-(iii)' are explicitly given). Recall that $L_{p},(1 \leq p \leq \infty)$, denotes the space of all measurable functions $f$ such that $|f|^{p}$ is integrable on $[0,1]$.

Theorem 2. Given a sequence $a_{n}, n=0,1,2, \ldots$, of complex numbers, there exists

(i) a function $f \in L_{1}$ such that $a_{n}$ are the moments of $f$;

(ii) a function $f \in L_{p}, 1<p \leq \infty$, such that $a_{n}$ are the moments of $f$;

(iii) a complex, regular Borel measure $\mu$ such that $a_{n}$ are the moments of $\mu$;

(iv) a nonegative regular Borel measure $\mu$ such that $a_{n}$ are the moments of $\mu$;

(v) a continuous function $f$ such that $a_{n}$ are the moments of $f$ if and only if the functions $L_{k}(t)\left\{a_{n}\right\}$

(i)' converge in the $L_{1}$-norm;

(ii)' are bounded in the $L_{p}$-norm;

(iii)' are bounded in the $L_{1}$-norm;

(iv)' are nonnegative;

(v)' converge uniformly.

For completeness, we give a little different and more compact proof of all parts using the following lemmas.

Lemma 3. For each $N$, define the linear map $T_{N}: L_{p} \rightarrow L_{p}$ (for all $p, 1 \leq$ $p \leq \infty)$ by

$$
\left(T_{N} g\right)(t)=\int_{0}^{1} K_{N}(t, s) g(s) \mathrm{d} s, \quad g \in L_{p} .
$$

Then each $T_{N}$ is continuous for all $p, 1 \leq p \leq \infty$, and there exists a constant $D<\infty$ such that $\left\|T_{N}\right\| \leq D$ for all $N$. 


\section{MOMENTS OF VECTOR-VALUED FUNCTIONS AND MEASURES}

P r o of. When $p=1$, we have, by Lemma l, for each $g \in L_{1}$,

$$
\begin{aligned}
\sup _{N}\left\|T_{N} g\right\| & =\sup _{N} \int_{0}^{1}\left|\int_{0}^{1} K_{N}(t, s) g(s) \mathrm{d} s\right| \mathrm{d} t \\
& \leq \sup _{N} \int_{0}^{1}\left(|g(s)| \int_{0}^{1}\left|K_{N}(t, s)\right| \mathrm{d} t\right) \mathrm{d} s \leq M\|g\| .
\end{aligned}
$$

Therefore $\sup \left\|T_{N}\right\|=D_{1}<\infty$. When $p=\infty$, for each $N$,

$$
\begin{aligned}
\left\|T_{N}\right\| & =\sup _{\|g\|=1} \operatorname{ess} \sup _{t}\left|\int_{0}^{1} K_{N}(t, s) g(s) \mathrm{d} s\right| \\
& \leq \sup _{\|g\|=1} \operatorname{ess} \sup _{t} \int_{0}^{1}\left|K_{N}(t, s) \| g(s)\right| \mathrm{d} s=D_{2}<\infty .
\end{aligned}
$$

Putting $D=\max \left(D_{1}, D_{2}\right)$, we have that, for each $N, T_{N}$ is a linear mapping of each $L_{p}$ space into itself $(1 \leq p \leq \infty)$ and $T_{N}$ is continuous for $p=1$ and $p=\infty$ with norm at most $D$. The result now follows from the Riesz convexity theorem [E, p. 526].

Lemma 4. If $f$ is in $L_{p}([0,1]), 1 \leq p<\infty$, then $T_{N} f$ converges to $f$ in the $L_{p}$-norm.

P r o of. Let $\epsilon>0$ be given. Then there exists $\psi \in C([0,1])$ such that $\|f-\psi\|_{p}<\epsilon$. So, for all sufficiently large $N$,

$$
\begin{aligned}
\left\|T_{N} f-f\right\| & \leq\left\|T_{N}(f-\psi)\right\|+\left\|T_{N} \psi-\psi\right\|+\|\psi-f\| \\
& \leq\left\|T_{N}\right\|\|f-\psi\|+\epsilon+\epsilon \\
& <D \epsilon+2 \epsilon .
\end{aligned}
$$

Lemma 5. If $f$ is in $L_{\infty}([0,1])$, then $T_{N} f$ is a bounded subset of $L_{\infty}([0,1])$ and so weak-star compact and (some subsequence of) $T_{N} f$ converges to $f$ in the weak-star topology of $L_{\infty}$.

Pr o of. For all $f$ in $L_{\infty}([0,1])$ the sequence $\left(T_{N} f\right)$ is bounded in $L_{\infty}([0,1])$, and so weak-star compact.

Proof of Theorem 2. Suppose that $f$ is in $L_{1}$ and $a_{n}$ are the moments of $f$. Then $L_{N}=T_{N} f$ and so (i)' follows from Lemma 4. Also if $f$ is in $L_{p}$, (ii)' follows from Lemma $4(1<p<\infty)$ and Lemma $5(p=\infty)$. 


\section{MILOSLAV DUCHOŇ — CAMILLE DEBIÈVE}

If the $a_{n}$ are the moments of a complex measure $\mu, a_{n}=\int_{0}^{1} s^{n} \mu(\mathrm{d} s)$ then, by Lemma 1

$$
\begin{aligned}
\left\|L_{N}\right\|_{1}=\int_{0}^{1}\left|L_{N}(t)\right| \mathrm{d} t & =\frac{N+1}{N} \sum_{m=0}^{N-1}\left|\lambda_{N, m}\right| \\
& \leq \frac{N+1}{N} \sum_{m=0}^{N}\left(\begin{array}{l}
N \\
m
\end{array}\right) \int_{0}^{1} s^{m}(1-s)^{N-m}|\mu|(\mathrm{d} s) \\
& \leq M|\mu|([0,1])
\end{aligned}
$$

which shows that (iii)' follows from (iii).

We now show the converse implications. First, observe that, if $n$ is fixed and $N>n$, then

$$
\int_{0}^{1} t^{n} L_{N}(t) \mathrm{d} t \rightarrow a_{n}, \quad(n=0,1,2, \ldots), N \rightarrow \infty .
$$

If the $L_{N}$ converge in the $L_{1}$-norm, they converge to an integrable function $f$. So

$$
\begin{aligned}
\left|\int_{0}^{1}\left(f(t)-L_{N}(t)\right) t^{n} \mathrm{~d} t\right| & \leq \int_{0}^{1}\left|f(t)-L_{N}(t)\right|\left|t^{n}\right| \mathrm{d} t \\
& \leq \sup _{t}\left|t^{n}\right|\left\|f-L_{N}\right\|_{1} .
\end{aligned}
$$

Therefore,

$$
\lim _{N}\left|\int_{0}^{1} f(t) t^{n} \mathrm{~d} t-\int_{0}^{1} L_{N}(t) t^{n} \mathrm{~d} t\right|=0
$$

Hence, by (3),

$$
\int_{0}^{1} f(t) t^{n} \mathrm{~d} t=a_{n}
$$

Suppose that $L_{N}$ are bounded in $L_{p}$-norm, $1<p \leq \infty$. We may as well assume that $\left\|L_{N}\right\|_{p} \leq 1, N=1,2, \ldots$ Then the $L_{N}$ lie in the unit ball of the conjugate space of $L_{q}$ (where $p^{-1}+q^{-1}=1$ ). Since this unit ball is weak-star compact, there is a function $f$ in $L_{p}$ with $\|f\|_{p} \leq 1$ such that every weak-star neighbourhood of $f$ contains $L_{N}$ for infinitely many values of $N$. In other words, 


\section{MOMENTS OF VECTOR-VALUED FUNCTIONS AND MEASURES}

given any $g$ in $L_{q}$, the numbers

$$
\int_{0}^{1} L_{N}(t) g(t) \mathrm{d} t
$$

are near $\int_{0}^{1} f(t) g(t) \mathrm{d} t$ for infinitely many values of $N$. But each $t^{n}$ is in $L_{q}$ and so, by (3)

$$
\int_{0}^{1} f(t) t^{n} \mathrm{~d} t=a_{n}
$$

Suppose that

$$
\int_{0}^{1}\left|L_{N}(t)\right| \mathrm{d} t \leq \alpha
$$

for all $N$. Define, for each $N$, the scalar-valued map $\Phi_{N}$ on $C([0,1])$ by

$$
\Phi_{N}(\psi)=\int_{0}^{1} \psi(t) L_{N}(t) \mathrm{d} t, \quad \psi \in C([0,1]) .
$$

Then, for all $N,\left\|\Phi_{N}\right\| \leq \alpha$. That is, all the $\Phi_{N}$ are in the (weak-star compact) ball of radius $\alpha$ of the dual space of $C([0,1])$. Hence there exists a regular Borel measure $\mu$ such that, for all $\epsilon>0$ and all $\psi \in C([0,1])$, there exists $N$ such that

$$
\left|\int_{0}^{1} \psi(t) L_{N}(t) \mathrm{d} t-\int_{0}^{1} \psi(t) \mu(\mathrm{d} t)\right|<\epsilon .
$$

Since each $t^{n}$ is in $C([0,1])$, we have, by (3),

$$
\int_{0}^{1} t^{n} \mu(\mathrm{d} t)=a_{n}
$$

Remark. By using $\lim _{N \rightarrow \infty}\left\|L_{N}-f\right\|_{1}=0$, in conjunction with results in general integration theory referring to criteria for weak compactness for subsets of $L_{1}$ (see [DS, pp. 294-295] and [E, pp. 274-276]) the following characterization of $L_{1}$ moments can be established.

Recalling an operator $L_{N}(t)$ defined by

$$
L_{N}(t)=(N+1) l_{N,[N t]} \quad(k=1,2, \ldots ; 0 \leq t \leq 1)
$$

the following four conditions are equivalent: 


\section{MILOSLAV DUCHOŇ — CAMILLE DEBIÈVE}

(a) a subsequence $\left(L_{N_{k}}\right)_{k=1}^{\infty}$ exists such that the set functions $E \rightarrow \int_{E} L_{N_{k}}(t) \mathrm{d} t$ $(k=1,2, \ldots)$ are uniformly (or equi-) absolutely continuous, that is, to each $e>0$ there corresponds $d>0$ such that

$$
\sup _{k}\left|\int_{E} L_{N_{k}}(t) \mathrm{d} t\right| \leq e
$$

for all measurable sets $E$ satisfying $m(E)<d$;

(b) some subsequence $\left(L_{N_{k}}\right)_{k=1}^{\infty}$ converges weakly in $L^{l}$;

(c) some subsequence $\left(L_{N_{k}}\right)_{k=1}^{\infty}$ converges in $L^{1}$;

(d) $\left(a_{n}\right)_{n \in Z} \in M o(Z)$, i.e., $a=M o f, f \in L^{1}$, Mo denotes the moment.

If any one of these conditions holds, and if $f=\lim _{k} L_{N_{k}}$ weakly or strongly in $L^{1}$, then $a=\operatorname{Mof}, L_{N}=L_{N} f$, and so $\lim _{N \rightarrow \infty}\left\|L_{N}-f\right\|_{1}=0$.

Remark. The uniform absolute continuity of the set functions can be expressed as uniform absolute continuity of the point functions

$$
F_{k}(x)=\int_{0}^{x} L_{N_{k}}(t) \mathrm{d} t
$$

the condition being precisely that to each $e>0$ there corresponds a number $d=d(e)>0$ such that

$$
\sup _{k} \sum_{j=1}^{r}\left|F_{k}\left(b_{j}\right)-F_{k}\left(a_{j}\right)\right| \leq e
$$

for any finite sequence $\left(\left(a_{j}, b_{j}\right)\right)_{j=1}^{r}$ of disjoint open intervals $\left(a_{j}, b_{j}\right)$ the sum of whose lengths does not exceed $d$. In this connection see, for example, HS, Theorem (19.53) and its proof].

\section{Vector-valued moments}

Let $X$ be a Banach space. Note that at some places, e.g., in Condition A the absolute value will be replaced by norm in $X$.

TheORem 6. Given a sequence $a_{n}, n=0,1,2, \ldots$ of elements of $X$, there exists a regular measure $\mu: \mathcal{B}([0,1]) \rightarrow X$ of finite total variation such that $a_{n}$ are the moments of $\mu$ if and only if there exists a constant $0<H<\infty$ such that

$$
\int_{0}^{1}\left\|L_{N}(t)\right\| \mathrm{d} t \leq H, \quad N=1,2, \ldots
$$




\section{MOMENTS OF VECTOR-VALUED FUNCTIONS AND MEASURES}

P r o of. Suppose that such a measure exists. Then, for each $N$, by (1)

$$
\begin{aligned}
\int_{0}^{1}\left\|L_{N}\right\| \mathrm{d} t & =\int_{0}^{1}\left\|\int_{0}^{1} K_{N}(t, s) \mu(\mathrm{d} s)\right\| \mathrm{d} t \\
& \leq \int_{0}^{1} \int_{0}^{1}\left|K_{N}(t, s)\right| \mathrm{d} t|\mu|(\mathrm{d} s) \\
& \leq M|\mu|([0,1]) .
\end{aligned}
$$

Converse1y, suppose that $\int_{0}^{1}\left\|L_{N}\right\| \mathrm{d} t \leq H$ for all $N$. If we define

$$
\Phi_{N}(\psi)=\int_{0}^{1} \psi(t) L_{N}(t) \mathrm{d} t, \quad \psi \in \mathcal{C}([0,1]),
$$

then $\left\|\Phi_{N}\right\| \leq H$ for all $N$. Since, for each $n$, $\lim _{N} \Phi_{N}\left(t^{n}\right)=a_{n}$, we have that $\lim _{N} \Phi_{N}(\psi)$ exists for all $\psi$ which are linear combinations of the $t^{n}$ and so, as $\left\|\Phi_{N}\right\| \leq H$ for all $N$, we conclude that $\lim _{N} \Phi_{N}(\psi)$ exists for all $\psi \in$ $C([0,1])$. Denote this limit by $\Phi$. To obtain our required measure, we use the following lemma (see $[\mathrm{D}, \S 19$, pp. 380,383]). For each subset $A$ of $[0,1]$, let $C([0,1], A)$ denote the space of continuous functions on $[0,1]$ vanishing outside $A$. If $F: C([0,1]) \rightarrow X$ is a linear mapping, define for each $A$,

$$
\left\|F_{A}\right\|=\sup \sum\left\|F\left(\psi_{i}\right)\right\|,
$$

where supremum is over all finite families $\psi_{i}$ in $C([0,1])$ with $\sum\left|\psi_{i}(t)\right| \leq \chi_{A}(t)$ for all $t \in[0,1]$.

Lemma. If $F: C([0,1]) \rightarrow X$ is a linear mapping, then there exists a reqular measure $\mu: \mathcal{B}([0,1]) \rightarrow X$ with finite variation such that

$$
F(\psi)=\int_{0}^{1} \psi(t) \mu(\mathrm{d} t), \quad \psi \in C([0,1]),
$$

if and only if $\left\|\left|F_{A} \|\right|<\infty\right.$ for all $A \in \mathcal{B}([0,1])$.

Let $A$ be in $\mathcal{B}([0,1])$ and let $\left\{\psi_{i} ; i=1,2, \ldots, n\right\}$ be a finite family of functions in $C([0,1], A)$ with

$$
\sum_{i=1}^{n}\left|\psi_{i}(t)\right| \leq \chi_{A}(t), \quad t \in[0,1]
$$

Then for each $N$,

$$
\sum_{i=1}^{n}\left|\psi_{i}(t)\right| \leq \chi_{A}(t), \quad t \in[0,1]
$$


So for each $N$,

$$
\begin{aligned}
\sum_{i=1}^{n}\left\|\Phi_{N}\left(\psi_{i}\right)\right\| & =\sum_{i=1}^{n}\left\|\int_{0}^{1} \psi_{i}(t) L_{N}(t) \mathrm{d} t\right\| \\
& \leq \sum_{i=1}^{n} \int_{0}^{1}\left|\psi_{i}(t)\right|\left\|L_{N}(t)\right\| \mathrm{d} t \\
& \leq \int_{0}^{1} \chi_{A}(t)\left\|L_{N}(t)\right\| \mathrm{d} t \leq H .
\end{aligned}
$$

Hence $\sum_{1}^{n}\left\|\Phi\left(\psi_{i}\right)\right\| \leq H$ and so $\left\|\left|\Phi_{A} \|\right| \leq H\right.$. Therefore, there exists a regular measure $\mu: \mathcal{B}([0,1]) \rightarrow X$ with finite variation such that

$$
\Phi(\psi)=\int_{0}^{1} \psi(t) \mu(\mathrm{d} t), \quad \psi \in C([0,1]) .
$$

But each $t^{n}$ is in $C([0,1])$ and so,

$$
a_{n}=\int_{0}^{1} t^{n} \mu(\mathrm{d} t)
$$

and thus theorem is proved.

Recall a definition of a Bochner integrable function. If $f:[a, b] \rightarrow X$ is simple, i.e., $f(s)=\sum_{i=1}^{n} \chi_{E_{i}}(s) x_{i}$, where $\chi_{E}$ denotes the indicator function of the set $E \subset[a, b], x_{i} \in X$, then for any $E \in B([a, b])$

$$
\int_{E} f \mathrm{~d} \lambda=\sum_{i=1}^{n} \lambda\left(E \cap E_{i}\right) x_{i}
$$

where $\lambda$ is a probability measure on $[a, b]$. Such functions are $\lambda$-measurable. Any function $f:[a, b] \rightarrow X$ which is the $\lambda$-almost everywhere limit of a sequence of simple functions is (called) $\lambda$-measurable.

A $\lambda$-measurable function $f:[a, b] \rightarrow X$ is called Bochner integrable if there exists a sequence of simple functions $\left(f_{n}\right)$ such that

$$
\lim \int_{[a, b]}\left\|f_{n}(s)-f(s)\right\| \mathrm{d} \lambda(s)=0 .
$$




\section{MOMENTS OF VECTOR-VALUED FUNCTIONS AND MEASURES}

In this case $\int_{E} f \mathrm{~d} \lambda$ is defined for each measurable set $E$ in $[a, b]$ by

$$
\int_{E} f \mathrm{~d} \lambda=\lim _{n} \int_{E} f_{n} \mathrm{~d} \lambda .
$$

A $\lambda$-measurable function $f:[a, b] \rightarrow X$ is Bochner integrable if and only if $\int_{[a, b]}\|f\| \mathrm{d} \lambda<\infty$.

The following theorem concerns moments of Bochner integrable function.

Theorem 7. Given a sequence $a_{n}, n=0,1,2, \ldots$, of elements of $X$, there exists an $X$-valued Bochner integrable function $f$ on $[0,1]$ such that $a_{n}$ are the moments of $f$ if and only if

$$
\lim _{N, J \rightarrow \infty} \int_{0}^{1}\left\|L_{N}(t)-L_{J}(t)\right\| \mathrm{d} t=0 .
$$

P r o of. Suppose that $f$ is Bochner integrable and $a_{n}$ are the moments of $f$. Let $\left\{A_{i}\right\}_{1}^{n}$ be a finite family in $\mathcal{B}([0,1])$ and $\left\{\beta_{i}^{n}\right\}$ a finite family of vectors in $X$ and define $g:[0,1] \rightarrow X$ by

$$
g(t)=\sum_{i=1}^{n} \beta_{i} \chi_{i}(t)
$$

Then

$$
\begin{aligned}
\int_{0}^{1}\left\|\int_{0}^{1} K_{N}(t, s) g(s) \mathrm{d} s-g(t)\right\| \mathrm{d} t & =\int_{0}^{1}\left\|\sum_{i=1}^{n} \beta_{i}\left(\int_{0}^{1} K_{N}(t, s) \chi_{A_{i}}(s) \mathrm{d} s-\chi_{A_{i}}(t)\right)\right\| \mathrm{d} t \\
& \leq \sum_{i=1}^{n}\left(\left\|\beta_{i}\right\| \int_{0}^{1}\left|\int_{0}^{1} K_{N}(t, s) \chi_{A_{i}}(s) \mathrm{d} s-\chi_{A_{i}}(t)\right| \mathrm{d} t\right)
\end{aligned}
$$

which, by Lemma 4 , tends to 0 as $N \rightarrow \infty$. Therefore, since the set of all such $g$ is dense in the space of all Bochner integrable functions and as

$$
L_{N}(t)=\int_{0}^{1} K_{N}(t, s) f(s) \mathrm{d} s,
$$

we have

$$
\lim _{N} \int_{0}^{1}\left\|L_{N}(t)-f(t)\right\| \mathrm{d} t=0 .
$$

Conversely, suppose that the sequence $L_{N}$ is Cauchy in the norm of the space of all Bochner integrable functions. Since this space is Banach, $L_{N}$ converges in the Bochner space norm to a Bochner integrable function $f$. 


\section{MILOSLAV DUCHOŇ — CAMILLE DEBIÈVE}

So, for each $n$,

$$
\begin{aligned}
\left\|\int_{0}^{1}\left(f(t)-L_{N}(t)\right) t^{n} \mathrm{~d} t\right\| & \leq \int_{0}^{1}\left\|f(t)-L_{N}(t)\right\|\left|t^{n}\right| \mathrm{d} t \\
& \leq \sup _{t}\left|t^{n}\right|\left\|L_{N}-f\right\|_{B} \rightarrow 0, \quad N \rightarrow \infty .
\end{aligned}
$$

Thus, by (3),

$$
a_{n}=\int_{0}^{1} t^{n} f(t) \mathrm{d} t .
$$

So the theorem is proved.

\section{REFERENCES}

[BDS] BARTLE, R.-DUNFORD, N.-SCHWARTZ, J. C.: Weak compactness and vector measures, Canad. J. Math. 7 (1955), 289-305.

[D] DINCULEANU, N.: Vector Measures. Pergamon Press, New York, 1967.

[DS] DUNFORD, N.-SCHWARTZ, J. T.: Linear Operators. Part I, in: Pure Appl. Math. (N.Y.), Vol. 6, Wiley-Intersci., New York, 1958.

[E] EDWARDS, R. E.: Functional Analysis: Theory and Applications. Holt Reinehart and Winston, Inc., New York, 1965.

[H] HAUSDORFF, F.: Summations Methoden und Momentenfolgen II, Math. Z. 9 (1921), 280-299.

[HS] HEWITT, E.-STROMBERG, K.: Real and Abstract Analysis. Springer-Verlag, Berlin, 1965.

[Lo] LOREnTZ, G. G.: Bernstein Polynomials. Toronto University Press, Toronto, 1953.

[W] WIDDER, D. V.: The Laplace Transform. Princetom University Press, Princeton, N. J., 1946.

Received December 17, 2008

Miloslav Duchoñ

Mathematical Institute

Slovak Academy of Sciences

Štefánikova 49

SK-814-73 Bratislava

SLOVAKIA

E-mail: duchon@mat.savba.sk

Camille Debiève

Université Catholique de Louvain

Département de Mathématiques

2, Chemin du Cyclotron

B-1348 Louvain-la-Neuve

BELGIUM

E-mail: camille.debieve@uclouvain.be 